\title{
ITALIANOS EN LA ADMINISTRACIÓN TERRITORIAL ESPAÑOLA DEL SIGLO XVIII
}

\author{
María del Carmen IRLES VICENTE \\ Universidad de Alicante
}

\section{Resumen}

A lo largo del Setecientos fueron muchos los italianos que acudieron a España para desarrollar algún tipo de actividad económica o cultural, pero también los hubo que llegaron dispuestos a ocupar un puesto en la dirección del Estado; en el presente trabajo prescindimos de aquellos individuos que desempeñaron empleos en la administración central, para ocuparnos de quienes aplicaron las directrices de la monarquía borbónica a nivel territorial: capitanes generales, intendentes, corregidores y gobernadores.

\begin{abstract}
Many were the Italians who attended to Spain for developing some type of economic or cultural activity all along the 18 th Century, but also there were some who arrived prepared to occupy any post in the direction of the State. In the present report we dispensed those who were employed by the central administration, an so focus on those who applied the norms of the Bourbonic monarchy at a territorial level: general captains, intendants, «corregidores» and governors.
\end{abstract}

La tradicional vinculación existente entre España e Italia desde la Edad Media -en principio como consecuencia de las conquistas practicadas por los reyes de la Corona de Aragón; afianzada más tarde, ya en la Edad Moderna, merced a la política desarrollada por los Austrias; y, finalmente, condicionada por los intereses de los Borbones, ya en el XVIII- favoreció la incorporación de buen número de italianos a la administración española setecentista '. La composición plurinacional del ejército ${ }^{2}$, y la cos-

1. Sobre la presencia de italianos en la España del Setecientos vid. PRADELLS NADAL, J., «Italianos en la España del siglo XVIIl», en GIMÉNEZ, E., LOZANO, M.A. y RÍOS, J.A., Españoles en Italia e italianos en España. IV Encuentro de investigadores de las universidades de Alicante y Macerata (mayo, 1995). Alicante, 1996, pp. 61-75. El análisis de un sector concreto, el de aquellos individuos que se hicieron cargo de la administración del Estado al más alto nivel, en OZANAM, D., «Les étrangers dans la 
tumbre de premiar con cargos de índole política a aquéllos que más se habían significado en su dirección, coadyuvó, asimismo, a que gentes nacidas en la península itálica o sus islas adyacentes asumieran empleos de gobierno en la España de las Luces.

Una buena muestra de la confianza que a los monarcas españoles del Setecientos $y$, en especial, a sus ministros inspiraban los sujetos nacidos en Italia queda perfectamente de manifiesto en las frases que el duque de Montemar dirigió al secretario de Estado y del Despacho Sebastián de la Cuadra en $1737^{3}$. Montemar, que acababa de dejar el empleo de capitán general de Cataluña para hacerse cargo de la Secretaría de Guerra, y por lo tanto era un buen conocedor de la realidad catalana, no dudaba en proclamar que:

«El Gobierno de Barcelona es el de la primera confianza de España, y siempre S.M. lo ha conferido a los oficiales más graduados, antiguos y de más experiencias, como lo acredita el haber sido, después que se restauró aquella plaza, sus Gobernadores el Marqués de Lede, yo, D. Francisco Varix, y el Conde de Roydeville; en esta inteligencia he propuesto a S.M. al Teniente General Marqués de Pozoblanco, y a los Mariscales de Campo más antiguos de estos ejércitos (...), añadiendo a lo referido que estando encargado el mando de Cataluña al Conde de Glimes, flamenco, y el Gobierno de la Ciudadela al Marqués de Verboon, de la misma nación, encontré muy conveniente al servicio de S.M. que el de la Plaza de Barcelona recayese en un español o italiano para dejar consoladas estas dos naciones»".

No pretendemos con este artículo realizar un análisis exhaustivo de la larga nómina de italianos que ocuparon empleos de gobierno en la España de las Luces, sino únicamente presentar algunos ejemplos que pongan de relieve la importancia que dicho contingente tuvo, a nuestro entender, en la administración española setecentista, tanto a nivel civil como militar.

Por razones obvias de espacio, prescindimos en el presente trabajo de aquellos italianos que desempeñaron empleos en la administración central (secretarios de Estado como Esquilache o el duque de Grimaldi; consejeros de Guerra como Horacio Borghese o Domingo Giudice, príncipe de Cellamare; de Indias como Luis Yopulo y Spadafora; de Hacienda como Juan Brancacho, etc.), para centrarnos en quienes aplicaron las directrices de la monarquía borbónica a nivel territorial: capitanes generales, intendentes, corregidores y gobernadores.

La importancia que dentro del organigrama institucional borbónico alcanzaba la figura del capitán general impulsó a confiar este empleo a sujetos de la primera nobleza y que frecuentemente contaban con un título nobiliario. Ni qué decir tiene que fueron bastantes los italianos que llegaron a disfrutar de este empleo en diferentes distritos peninsulares a lo largo del siglo XVIII.

haute administration espagnole au XVIII' siècle», en Pouvoirs et société dans l'Espagne moderne. Hommage à Bartolomé Bennasar. Toulouse, 1993, pp. 215-229.

2. Sobre la composición plurinacional del ejército español en el Setecientos vid. ANDÚJAR CASTILLO, F., Los militares en la España del siglo XVIII. Un estudio social. Granada, 1991, pp. 313-325.

3. Sobre Sebastián de la Cuadra vid. ESCUDERO, J.A., Los orígenes del Consejo de Ministros en España, Madrid, 1979, vol. I, pp. 99 y ss.

4. A.G.S. Guerra Moderna. Leg. 1.887. El duque de Montemar a Sebastián de la Cuadra. Barcelona, 5 de septiembre de 1737. 
En el caso de la capitanía general de Valencia, por citar un ejemplo concreto, los titulares de origen italiano fueron mayoría durante el reinado de Felipe V, volviendo a reaparecer nuevamente, aunque con carácter esporádico, con Carlos IV ${ }^{5}$; los príncipes de Campoflorido y Monforte, y los duques de San Pedro y Castropignano fueron los encargados de aplicar la política borbónica en tierras valencianas en los comienzos y finales del siglo.

Francisco María Spínola, duque de San Pedro, fue el tercer capitán general de Valencia y el primero de los cuatro que, originarios de Italia, desempeñaron dicho empleo en este distrito levantino a lo largo del Setecientos.

La llegada del duque de San Pedro a Valencia se produjo en 1718, en sustitución de Melchor de Avellaneda, marqués de Valdecañas, prolongándose su estancia por espacio de tres años, hasta que en 1721 fue llamado a la Corte para ocupar el puesto de ayo del príncipe Carlos, encargo en el que le sorprendería la muerte en la primavera de 1727 , cuando contaba 68 años de edad ${ }^{6}$.

Como la mayor parte de la nobleza, el duque de San Pedro gozó de numerosas distinciones y empleos honoríficos, tales como la grandeza de España, caballero de la orden del Santo Espíritu y gentilhombre de cámara.

Tras la salida de Francisco María Spínola, otro italiano vino a hacerse cargo de la capitanía general valenciana. Natural de Sicilia, Luis Reggio Branciforte, príncipe de Campoflorido, desempeñó los cargos de general de las galeras de la mencionada isla y el mando militar de Guipúzcoa con anterioridad a su incorporación a la capitanía general de Valencia, a la que accedió en 1721, en un primer momento con carácter interino y, ya en propiedad, a partir de $1727^{7}$.

La interinidad de su posesión inicial llevó a la Cámara de Castilla a consultar con el monarca, a principios de 1722 , la conveniencia de que se le diera un documento acreditativo del empleo que desempeñaba, ya que, según exponía aquélla, se hallaba «sirviendo el Gobierno Político de Valencia y Presidencia de la Audiencia sin habérsele expedido por este Tribunal el título que le corresponde». La Resolución regia, sin embargo, dejó en el aire temporalmente la adopción de la medida propuesta por la Cámara al dictaminar, el 12 de abril de ese mismo año, que «sirva y continúe con el Despacho y orden que tiene, sin necesitar ni dársele otro alguno» ${ }^{8}$.

La estancia de Campoflorido en Valencia, que se alargó por espacio de 16 años, resultó en algunos momentos bastante incómoda debido al enfrentamiento personal que sostuvo con el intendente-corregidor Clemente de Aguilar durante prácticamente todo el tiempo que éste permaneció al frente del cargo, esto es, el bienio 1725-279.

5. MOLAS RIBALTA, P., «Militares y togados en la Valencia borbónica», en Actes du lir Colloque sur le Pays Valencien a l'époque moderne. Pau, 1980, p. 178.

6. Gaceta de Madrid, 27 de mayo de 1727.

7. Gaceta de Madrid, 23 de septiembre de 1727.

8. A.G.S. Gracia y Justicia. Lib. 1.564.

9. Aguilar ya había dado con anterioridad sobradas muestras de su difícil carácter, hasta el extremo que tanto en Mallorca como Cerdeña, destinos en los que había servido, fue calificado como «loco». Sobre el tema vid. ABBAD, F. y OZANAM, D., Les intendants espagnols du XVIIF siècle. Madrid, 1992, p. 46, n. 1 . 
El conflicto entre ambas autoridades, que en un principio pareció tener su origen en problemas de protocolo, se fue agudizando más y más con el paso del tiempo, llegando a desembocar en serias acusaciones de corrupción.

El resultado de la pugna entre Campoflorido y Aguilar se saldó siempre en favor del primero, merced a los importantes valedores con que aquél contaba en la Corte, así como el gran crédito que tenía ante el rey. El intendente tuvo una clara prueba de ese respaldo hacia el capitán general cuando recibió, a comienzos del verano de 1726, un escrito en el que se recriminaba su conducta y se le animaba a tratar al militar con más deferencia:

«habiendo llegado a noticia del rey ha excedido V.S. en algunas expresiones contrarias a la estimación de la persona y carácter del príncipe de Campoflorido, me manda S.M. prevenir a V.S. se corrija en lo adelante en semejante modo de proceder $"{ }^{10}$.

Lejos de acatar de forma sumisa la recomendación anterior, Clemente de Aguilar se dirigió acto seguido al secretario de Guerra, marqués del Castelar, para quejarse de las largas esperas que el capitán general le obligaba a soportar, antes de recibirle, cuando acudía a tratar con él de asuntos oficiales.

Como ya adelantamos, con el transcurso del tiempo las acusaciones de Aguilar hacia Campoflorido se hicieron más duras y frecuentes, llegando a denunciar que obtenía beneficios de consentir la práctica clandestina de juegos prohibidos. No vamos a detenernos a analizar la larga serie de episodios que enfrentaron al intendente con el capitán general, la Audiencia, el ayuntamiento de Valencia e, incluso, parte de las tropas acuarteladas en la ciudad -y como consecuencia de los cuales se optó por trasladar a Clemente de Aguilar a otro destino ${ }^{11}$, aspectos todos ellos que han sido perfectamente recogidos en otros trabajos ${ }^{12}$; por el contrario, abordaremos, si bien sucintamente, la posterior trayectoria profesional de su antagonista.

El príncipe de Campoflorido permaneció como capitán general de Valencia hasta mediados de 1737, año en que fue destinado a la embajada de Venecia ${ }^{13}$. Su estancia en territorio italiano fue, sin embargo, bastante breve, ya que a comienzos de 1740 era designado nuevo embajador extraordinario en la Corte de Francia ${ }^{14}$.

Junto a los importantes cargos políticos desempeñados por el siciliano Campoflorido, merecen ser reseñadas también las distinciones que obtuvo a título honorífico, como la grandeza de España honoraria, que le fue concedida en febrero de 1710, y declarada de primera clase en septiembre de $1727^{15}$. También mantuvo vinculación con algunas órdenes militares, como la de Santiago, de la que fue comendador, y la de Calatrava, a la que se incorporó como caballero ${ }^{16}$.

10. A.G.S. Guerra Moderna. Leg. 1.722. Reprensión a Clemente de Aguilar. Madrid, 19 de junio de 1726.

11. Tras su salida de Valencia Clemente de Aguilar pasó a desempeñar el corregimiento de Granada, en cuyo destino fallecería en agosto de 1733.

12. GIMÉNEZ LÓPEZ, E., Militares en Valencia (1707-1808). Alicante, 1990, pp. 128-134.

13. Gaceta de Madrid, 4 de junio de 1737.

14. Gaceta de Madrid, I de marzo de 1740.

15. ATIENZA, J., Nobiliario español. Madrid, 1948, p. 1.408.

16. MOLAS RIBALTA, P., «Militares y togados...», p. 178. 
Más de medio siglo después de que Campoflorido abandonara la ciudad del Turia, en 1801 fue nuevamente designado capitán general de Valencia un siciliano, el príncipe de Monforte; sin embargo, esta vez el interesado apenas si desempeñó el cargo por espacio de unos días, al ser cesado a mediados del mes de agosto tras acceder a las peticiones populares de suprimir la formación de unas milicias provinciales ${ }^{17}$. También por un tiempo limitado había ocupado ese empleo unos años antes el duque de Castropignano, aunque con carácter interino ${ }^{18}$.

La capitanía general de Valencia no fue la única en contar entre sus titulares con individuos venidos de Italia; también en Cataluña, Aragón, Castilla, Extremadura o Canarias el máximo cargo político-militar recayó en diferentes momentos en sujetos de dicha procedencia.

Nacido en 1725 en Nápoles, José Gregorio Mauro -hijo de Leopoldo Gregorio, futuro marqués de Esquilache y Vallesantoro ${ }^{19}$ - ingresó en el ejército como cadete en 1744, participando activamente en las guerras italianas. Cuando su padre marchó a la península ibérica, a la muerte de Fernando VI, José le acompañó, viéndose beneficiado también por esa especie de espiral del éxito que durante los primeros años sesenta rodeó a la familia Gregorio. Brigadier desde mediados de 1760, y mariscal de campo desde abril de 1763, un año más tarde se le confió el corregimiento de Gerona, en el que venía a sustituir al marqués de Alós y en el cual permaneció hasta que en 1772, dos años después de haber sido ascendido a teniente general, pasó a obtener la titularidad del gobierno barcelonés ${ }^{20}$.

La presencia de José Gregorio al frente del corregimiento de la capital catalana fue, sin embargo, efímera. Comandante general interino de Extremadura desde julio de $1773^{21}$, y capitán general de Castilla en propiedad desde noviembre de $1775^{22}$, en la vacante por ascenso del marqués de Camarasa a la capitanía general extremeña, durante el tiempo que permaneció en dicho destino se fundó la Sociedad Económica de Amigos del País de Zamora, que pasó a presidir desde su constitución en el año 1778.

De Zamora José Gregorio pasó en 1779 a hacerse cargo de la capitanía general de Aragón, falleciendo en dicho destino cinco años más tarde ${ }^{23}$.

Los dos hijos habidos del matrimonio entre el marqués de Vallesantoro y María Ignacia de Paternó, hija del capitán del regimiento de Palermo Mario Paternó, siguieron la carrera de las armas, si bien sólo el primogénito llegó a emular a su padre.

17. Ante los labradores congregados en la puerta de San Vicente de la capital valenciana el capitán general prometió la suspensión del sorteo para la formación de unas milicias provinciales. Cfr. ARDIT LUCAS, M., «Los alborotos de 1801 en el reino de Valencia», en Hispania 113 (1969), pp. 526-542, esp. p. 528 y $533-534$.

18. MOLAS RIBALTA, P., «Militares y togados...», p. 181.

19. Los servicios prestados por Leopoldo Gregorio al monarca napolitano -futuro Carlos III de Españafueron recompensados con la concesión del título de marqués de Esquilache en 1755.

20. Gaceta de Madrid, 15 de julio de 1760; 5 de abril de 1763; y 3 de abril de 1770. ESCARTÍN SÁNCHEZ, E., «El corregiment de Barcelona: notes per al seu estudi», en Primer Congrés d'Història Moderna de Catalunya. Barcelona, 1984. Vol. II, pp. 47-55.

21. Gaceta de Madrid, 27 de julio de 1773.

22. Gaceta de Madrid, 3 de octubre de 1775 .

23. Gaceta de Madrid, 17 de septiembre de 1784. 
La actuación de Leopoldo Gregorio Paternó durante el bloqueo de Gibraltar le valió ser ascendido a coronel a principios de 1783 . También fue su participación en otro hecho bélico, en este caso la defensa del presidio de Orán, el que le facilitó la consecución del grado de brigadier a finales de 1791. Promovido a mariscal de campo, a comienzos del mes de septiembre de 1795 se le confió el corregimiento de Tarragona, donde permaneció por espacio de tres años hasta que en mayo de 1798 pasó a hacerse cargo del gobierno militar y político de Barcelona ${ }^{24}$.

Comandante General interino de Cataluña en dos ocasiones, desde el 22 de agosto de 1799 al 2 de noviembre de ese mismo año, y del 16 al 28 de mayo de 1801 , a finales de 1802 se le concedió la llave de gentilhombre de Cámara con entrada.

Algunos años más tarde, en 1807, cuando ya disfrutaba el grado de teniente general, el marqués de Vallesantoro fue designado virrey y capitán general de Navarra, en sustitución del marqués de las Amarillas, quien acababa de ser promovido al Consejo de Guerra ${ }^{25}$.

Otros militares nacidos en Italia que llegaron a estar al frente de una capitanía general fueron los hermanos Tiburcio y Carlos Carraffa, quienes ocuparon las de Guipúzcoa y Costa de Granada, respectivamente; Antonio Giudice, príncipe de Cellamare, que se hizo cargo de la gallega; o Francisco Pignatelli, quien asumió la de la costa granadina en $1746^{26}$.

Como ocurría en el empleo de capitán general, también en el cuerpo de intendentes encontramos un cierto número de sujetos que, si bien nacidos en Italia, tras su venida a España se arraigaron fuertemente en la península, tanto a nivel económico como familiar.

Tras cursar sus estudios en el Seminario de nobles de Liorna, Jorge Astraudi entró en 1734 al servicio de José del Campillo, por aquel entonces intendente general del ejército español en Italia ${ }^{27}$.

Empleado en un primer momento en la contaduría del ejército, donde se encargó, sucesivamente, de los hospitales y del abastecimiento de víveres, Astraudi pasó a España a comienzos de 1737.

La llegada del florentino a la península ibérica coincidió con la incorporación de Campillo, su protector, a la intendencia aragonesa, a donde se dirigió también aquél para prestar sus servicios.

Cuando Campillo dejó Zaragoza y marchó a la Corte, en 1741, para dirigir la Secretaría de Estado y Hacienda, le acompañó en su viaje Jorge Astraudi, quien a mediados del año siguiente empezaba a prestar sus servicios como escribiente en dicha secretaría. Unos meses más adelante, y tras unir Campillo en su persona las secretarías de Guerra, Marina e Indias, Astraudi fue trasladado a la primera de ellas ${ }^{28}$.

24. GAY ESCODA, J.M., El corregidor a Catalunya. Tesis doctoral inédita, Barcelona, 1991, ff. 447 y 417-418.

25. Gaceta de Madrid, 1 de diciembre de 1807.

26. OZANAM, D., «Les étrangers dans la haute administration espagnole...», p. 223.

27. Sobre la trayectoria profesional de Campillo vid. el estudio preliminar de Dolores MATEOS DORADO al libro de CAMPILLO Y COSSÍO, J., Dos escritos políticos, Oviedo, 1993, pp. XI-XLVI.

28. ESCUDERO, J.A., Los origenes del Consejo de Ministros..., vol. I, pp. 111 y ss. 
La muerte de su valedor, en abril de 1743, aunque pudo demorar parcialmente los ascensos en su carrera, no resultó decisiva en la trayectoria profesional de Jorge Astraudi, que fue designado comisario de guerra en 1751, y a quien se encomendaron diferentes comisiones a partir de ese momento, entre las que cabe destacar la administración del canal de Castilla, tarea que le fue confiada en octubre de 1764.

Comisario ordenador desde finales de $1774^{29}$, poco después pasó a hacerse cargo de forma interina de la intendencia de Galicia, que había quedado vacante tras producirse el fallecimiento del marqués de Piedrabuena. La negativa manifestada por José Antonio Armona a tomar posesión del cargo ${ }^{30}$ alargó unos meses la permanencia de Astraudi en el destino gallego, que no dejó hasta marzo de 1777, momento en que obtuvo en propiedad otra intendencia: la de Toro.

Apenas un año pasó Jorge Astraudi en tierras toresanas, ya que en marzo de 1780 marchaba a Jaén para hacerse cargo de su intendencia, de la que salió, seis años más tarde, a servir la de Valladolid, esta vez junto con el corregimiento. Éste sería su último destino, ya que a finales de 1789 se jubilaba, con medio sueldo, «en atención a su edad y achaques ${ }^{3 !}$, falleciendo mediada la década de los noventa ${ }^{32}$.

Natural de San Pablo, en Nápoles, también el aspecto más significativo de la carrera de Tomás Pascual Ripoli es su vinculación a las intendencias, si bien parece que antes de ello sirvió como administrador principal de la lotería real de Cádiz, por cuya labor al frente de dicho empleo recibió los honores del Consejo de Hacienda.

Su presencia en Cádiz no sólo fue fructífera a nivel profesional; también lo fue a nivel personal, ya que allí contrajo matrimonio con Rafaela de Herrera y Cruzat, dama de la reina, y miembro de una importante familia que había servido a la monarquía desde diferentes puestos de la administración y el ejército.

A los méritos de su padre, Francisco Herrera y Navarro, militar de profesión, quien a la altura de 1741 se hallaba graduado de alférez de fragata, y que años más adelante serviría los gobiernos de Villanueva de los Infantes ${ }^{33}$ y Cieza ${ }^{3.4}$, ambos en la orden de Santiago, cabía añadir los de sus abuelos, tíos, hermanos y primo.

Francisco Manuel de Herrera y Suárez de Toledo, abuelo de Rafaela, era fiscal de la Casa de Contratación de Sevilla cuando recibió, en agosto de 1731, los honores de consejero de Indias; dos años más tarde volvió a lograr nuevos honores, esta vez del Consejo de Castilla ${ }^{35}$, así como el nombramiento de consejero de Guerra, cargo que no juró hasta 1736. Tras la reforma de este Consejo en $1744^{36}$, que suprimía las

29. Gaceta de Madrid, 13 de diciembre de 1774, p. 447.

30. José Antonio Armona y Murga fue designado intendente de Galicia en 1776 «en consideración al celo y acierto con que ha establecido en la isla de Cuba las nuevas contribuciones reales y la dirección de los correos marítimos». Cfr. Gaceta de Madrid, 21 de mayo de 1776, p. 182. Más datos sobre la expe riencia vital y profesional de este personaje en ARMONA Y MURGA, J.A., Noticias privadas de casa litiles para mis hijos (recuerdos del Madrid de Carlos III) (Ed. de J. ÁLVAREZ DE BARRIENTOS, E. PALACIOS FERNÁNDEZ y M.C. SÁNCHEZ GARCÍA), Madrid, 1989.

31. Gaceta de Madrid, 17 de noviembre de 1789.

32. ABBAD, F. y OZANAM, D., Les intendants espagnols..., p. 57.

33. Gaceta de Madrid, 8 de junio de 1751 .

34. Gaceta de Madrid, 7 de agosto de 1764.

35. Título en San Ildefonso, 8 de septiembre de 1733, en A.H.N. Consejos. Lib. 736.

36. ANDÚJAR CASTILLO, F., Consejo y Consejeros de Guerra en el siglo XVIII. Granada, 1996, pp. 53-63. 
plazas de consejeros togados, se incorporó al de Castilla ${ }^{37}$, donde permaneció hasta que le sobrevino la muerte en abril de $1752^{38}$.

Independientemente de la actividad a la que dedicaran sus vidas los hijos del referido consejero, la práctica totalidad de ellos se vinculó desde muy joven a la orden de Santiago. Tanto Francisco, padre de la esposa de Ripoli, como sus hermanos Antonio, José y Juan, se recibieron como caballeros de Santiago en $1741^{30}$; orden a la que se incorporarían, asimismo, algunos años más tarde Manuel y Francisco, dos de los cuñados de Ripoli ${ }^{\text {to }}$.

La vinculación a la orden de Santiago por parte de la familia Herrera no es el único hecho que merece nuestra atención. Retomando lo que fue el ámbito de actuación a nivel profesional vamos a ocuparnos de dos de sus miembros más sobresalientes: Juan de Herrera, tío de la esposa de Tomás Ripoli, y Francisco Manuel, sobrino de aquél.

Dedicado a la carrera de las Leyes, Juan Herrera y Navarro frecuentó desde muy pronto los estudios de abogados famosos, como Joaquín de Zúñiga y Tomás de Azpu$\mathrm{ru}$, con el fin de adquirir la necesaria práctica para poder incorporarse a la administración de justicia en alguna Audiencia o Chancillería. Sin embargo, antes que su nombre empezara a ser barajado por la Cámara de Castilla para cualquiera de los tribunales peninsulares, Juan de Herrera hizo sus pinitos en el campo de la docencia al ingresar como profesor de Leyes y Cánones en la Universidad de Sevilla.

Fue a la muerte de su padre, ocurrida, como comentamos, en la primavera de 1752, cuando el joven letrado -que estaba a punto de cumplir los treinta años-solicitó la concesión de una plaza en la Audiencia de Cataluña para poder así atender al mantenimiento de sus parientes más allegados. Refería Herrera en su memorial que "habiendo quedado su pobre familia de madre, dos tias ancianas e impedidas, un hermano inhábil, tres sobrinos huérfanos, sin otros once a quienes les hará gran falta el difunto [su padre]» ${ }^{+1}$, sólo si se le concedía el mencionado empleo podría él hacerse cargo del sostenimiento de tan dilatada, y necesitada, parentela.

Los favorables informes que sobre el pretendiente evacuó Diego de Rojas, gobernador del Consejo, debieron resultar concluyentes para el positivo resultado de la pretensión. En un escrito enviado al marqués del Campo Villar, el obispo de Calahorra definía a Herrera como:

«juicioso, de buenos modales y prendas (...); es de conocida suficiencia y literatura, y de muy ajustada conciencia» ${ }^{42}$.

37. Francisco Manuel Herrera se incorporó en 1744 al Consejo de Castilla para cubrir la vacante ocurrida por fallecimiento de Andrés González Barcia. Título en Aranjuez, 11 de junio de 1744, en A.H.N. Consejos. Lib. 736.

38. ANDÚJAR CASTILLO, F., Consejo y consejeros..., p. 218-219.

39. CADENAS Y VICENT, V., Caballeros de la orden de Santiago. Siglo XVIII. Madrid, 1978, vol. III, pp. 161-162.

40. Los dos cuñados de Ripoli se incorporaron como caballeros de Santiago en el año 1781, en CÁRDENAS PIERA, E., Caballeros de la orden de Santiago. Siglo XVIII. Madrid, 1994, vol. VI, p. 57.

41. A.G.S. Gracia y Justicia. Leg. 152. Mentorial de Juan Herrera.

42. A.G.S. Gracia y Justicia. Leg. 152. Diego, obispo de Calahorra, al marqués del Campo de Villar, 23 de abril de 1752 . 
Una plaza de alcalde del crimen en la Audiencia catalana podía ser un buen lugar para poner en práctica semejantes atributos, de ahí que fuera ese cargo el que se le confió en un decreto fechado el 27 de abril de 1752.

Cuando ocho años más tarde se solicitaron informes sobre los ministros que integraban la Audiencia de Cataluña, el regente, Isidro de la Hoz, dijo que Juan de Herrera «discurre con variedad a los demás y con ninguno de los compañeros se trata» ${ }^{43}$. El obispo de Barcelona, por su parte, indicó que era «de literatura más que bastante, juicio vivo, penetrante y recto, de conducta irreprensible, amante del trabajo, de celo eficaz en el cumplimiento de las obligaciones de su ministerio ${ }^{44}$. Esta última opinión fue convenientemente recogida por la Cámara de Castilla, en la primavera de 1761, al proponerle, en segundo lugar, para ascender a oidor del tribunal catalán ${ }^{45}$.

No logró Herrera ser designado en esa ocasión, ni tampoco tres años más tarde, cuando nuevamente fue propuesto, esta vez encabezando la correspondiente terna, debiendo permanecer como alcalde del crimen hasta octubre de 1769 , momento en que pasó a cubrir la plaza de oidor que acababa de quedar vacante por fallecimiento de Alonso González de León ${ }^{46}$. Éste fue el último empleo que obtuvo Herrera, quien, pese a permanecer por espacio de casi dos décadas desempeñándolo, no promocionó a otro tribunal, finalizando sus días, y su presencia en la Audiencia catalana, en 1787.

Continuador, en cierta medida, de los pasos de su tío, Francisco Manuel Herrera, sobrino del magistrado al que acabamos de referirnos y primo de la esposa de Tomás Ripoli ${ }^{+7}$, cursó sus estudios de Jurisprudencia en la universidad de Granada. Concluidos éstos, en abril de 1772 se recibió como abogado en la Chancillería andaluza, poco antes de incorporarse a la docencia como catedrático regente de Sexto y Clementinas en la universidad donde había estudiado, actividad que desarrolló a lo largo de 1773.

También frecuentó Francisco Manuel algunas Academias de teórica y práctica, a las cuales asistió por espacio de cuatro años para mejorar su instrucción y adquirir la necesaria experiencia en la aplicación del Derecho.

Aunque desde un puesto más modesto que su tío y abuelo, también Francisco Manuel Herrera se incorporó a la administración del Estado, esta vez en su Andalucía natal. Tras ser consultado a lo largo de 1776 para la vara de Motril y la de Almuñécar y Salobreña, se le confió esta última ${ }^{48}$, permaneciendo en dicho empleo durante el preceptivo trienio. Concluido el período de ejercicio, sin embargo, tanto el propio interesado ${ }^{49}$, como el síndico personero Manuel Jiménez Vélez, y Miguel de Uriarte y Borja, aviador del ingenio de azúcares de la ciudad de Almuñécar, solicitaron que se le prorrogase en dicha vara, petición que fue desaconsejada por la Cámara en aten-

43. A.G.S. Gracia y Justicia. Leg. 590. Isidro de la Hoz al Marqués del Campo Villar. Barcelona, 29 de diciembre de 1759.

44. A.G.S. Gracia y Justicia. Leg. 590. El Obispo de Barcelona al Marqués del Campo Villar (1760).

45. A.G.S. Gracia y Justicia. Leg. 158. La Cámara, II de abril de 1761, propone para una plaza de oidor de la Audiencia de Cataluña.

46. A.G.S. Gracia y Justicia. Lib. 1.573; y A.G.S. Gracia y Justicia. Leg. 165. Plazas despachadas en El Escorial el 13 de octubre de 1769.

47. Francisco Manuel Herrera era hijo de Antonio Herrera y Navarro,

48. A.G.S. Gracia y Justicia. Leg. 161. La Camara 23 de octubre de 1776; también A.G.S. Gracia y Justicia. Lib. 1.575, y Gaceta de Madrid, 10 de diciembre de 1776, p. 443.

49. A.G.S. Gracia y Justicia. Lib 1.576. 
ción al informe redactado por la Chancillería de Granada, y «teniendo presente la oposición que hizo la sociedad, y lo expuesto por el fiscal» ${ }^{50}$.

Desconocemos cuáles fueron los pasos seguidos por Herrera tras concluir su labor en Almuñécar, pues aunque fue consultado a mediados de noviembre de 1781 para el corregimiento de Bujalance, también en Andalucía, lo cierto es que no logró la designación ${ }^{5 !}$.

Si buena parte de los Herrera dedicaron sus vidas a servir al rey al frente de un gobierno, corregimiento o Audiencia, como en toda buena familia que se preciase de serlo, tampoco faltan en ésta individuos vinculados a la Iglesia, como Manuel, canónigo de Granada ${ }^{52}$.

También los ascendientes por línea materna de Rafaela Herrera y Cruzat, esposa, como dijimos, de Tomás Pascual Ripoli, presentaban un perfil bastante similar al de la rama paterna, si bien en este caso su dedicación profesional les había llevado fuera de la península ibérica. Rafaela era nieta de Juan Cruzat y Góngora, marqués de Góngora, y bisnieta de Fausto Cruzat, un caballero de la orden de Santiago que fue gobernador y capitán general de Filipinas y presidente de su Audiencia ${ }^{53}$. Entre sus ascendientes figuraba, asimismo, Gervasio Cruzat y Góngora, un militar que fue destinado en la primavera de 1730 a América para hacerse cargo del gobierno de las Provincias de Nuevo Méjico ${ }^{54}$.

Comentado el ventajoso matrimonio contraído por Tomás Ripoli, así como el ámbito de actuación de la familia con la que emparentó, volvamos a los hitos más relevantes de su carrera. Incorporado en febrero de 1795 a la intendencia de Guadalajara, cuando dejó ese destino, en el invierno siguiente, fue para trasladarse a Córdoba, donde se mantuvo, igualmente, por espacio de un año, siendo designado esta vez para ocupar la intendencia de Galicia. Tampoco se alargó su estancia en tierras gallegas, al ser admitida su jubilación a finales de 1799.

Un último aspecto a destacar es la vinculación que, como sucediera con los familiares de su esposa, o con su propio hermano Domingo ${ }^{55}$, se estableció entre Tomás Ripoli y las órdenes militares, en este caso con la de Carlos III, a la que se incorporaba en $1792^{56}$.

El marcado carácter militar de los corregimientos implantados en tierras de la antigua Corona de Aragón a raíz de la Nueva Planta ${ }^{57}$, por un lado, y la composición plurinacional del ejército borbónico, por otro, contribuyeron a que fuera precisamente en este área del levante peninsular donde mayor número de italianos encontraron acomodo en un empleo como el de gobernador o gobernador-corregidor. El teniente co-

50. A.G.S. Gracia y Justicia. Leg. 163. La Cámara a 21 de agosto de 1782.

51. A.G.S. Gracia y Justicia. Leg. 163. La Cámara a 14 de noviembre de 1781.

52. Manuel Herrera era tío de la mujer de Tomás Ripoli.

53. CÁRdenAS PIERA, E., Caballeros de la orden de Santiago. Siglo XVIII. Madrid, 1994, vol. VI, p. 58.

54. Gacela de Madrid, 25 de abril de 1730.

55. Domingo Ripoli se incorporó a la orden de Santiago en 1779. Cfr. CÁRDENAS PIERA, E., Caballeros de la orden de Santiago. Siglo XVIII. Madrid, 1994, vol. VI, p. 46.

56. CADENAS Y VICENT, V., Caballeros de la Orden de Carlos III. Madrid, 1987, vol. XI, pp. 21-22.

57. En menor medida en los aragoneses. 
ronel Pedro Bonafede, corregidor de Orihuela desde 1776 hasta que le sobrevino la muerte en $1787^{58}$; o el brigadier Juan Antonio Caracciolo, que lo fue de Peñíscola entre 1749 y $1750^{5 y}$, momento en que presentó su dimisión, son sólo algunos de los numerosos ejemplos que se pueden citar.

Hortensio Domicio era un militar incorporado al ejército en 1737, que después de algunos años empuñando las armas fue designado gobernador de Benasque, en el Pirineo aragonés, empleo que desempeñó hasta 1765. Poco antes de concluir sus tareas en dicho destino, Domicio remitió un memorial al rey solicitando la concesión del corregimiento de Huesca, donde pretendía seguir dando muestras de su dedicación al real servicio. Sólo parcialmente fueron atendidos los deseos del capitán Domicio, ya que si bien se le confío el cargo de corregidor no fue en la ciudad solicitada, sino en Cervera, en el principado de Cataluña ${ }^{6}$. Allí concluyó sus días, el 9 de marzo de 1777 , sin obtener el deseado ascenso a teniente coronel, que había pretendido en abril de 1773, ni tampoco su paso a otro corregimiento de mayor relieve.

Después de medio siglo participando en todos los acontecimientos bélicos ocurridos, Antonio Labeli, un brigadier que llevaba en el ejército desde 1711, solicitó el corregimiento de Talarn alegando las muchas heridas recibidas, así como la enfermedad que padecía en las manos, la cual le dejaba enteramente inútil para el servicio. El rey, para premiar su dedicación, no dudó en recompensarle con el empleo apetecido ${ }^{61}$.

Cumplido el trienio en tierras catalanas, Labeli fue trasladado a Teruel; sin embargo, y pese a reconocer que era mejor destino que el que se hallaba sirviendo, el viejo militar pidió ser eximido del mismo en atención a que:

«pone a riesgo su vida en la mudanza por hallarse lleno de heridas y achaques, de avanzada edad; el camino largo, y por lo montuoso precisado a hacer el viaje a caballo, que no lo podría conseguir por estar estropeado de las manos, brazos y cabeza; y el clima de Teruel muy contrario a sus heridas por lo frío».

Pese al lamentable estado en que se encontraba, Antonio Labeli todavía hacía galas de una ciega obediencia al rey al señalar «que si V.M., sin embargo de lo referido, fuese servido mandar que se ejecute su Real determinación, marchará a pie las 8 jornadas que hay desde Talarn a Teruel, sacrificándose gustoso en servicio de V.M..$^{62}$.

Aunque desconocemos si llegó a producirse una respuesta por parte del monarca, ya fuera en un sentido u otro, lo cierto es que tampoco hizo falta, ya que Labeli falleció poco después en Talarn.

«En vista de tantos que de su clase, graduación y servicios han sido colocados y promovidos», el teniente coronel Jerónimo Gentile se aprestó a solicitar en 1766 el corregimiento que acababa de dejar vacante su compatriota Labeli, así como el grado de coronel ${ }^{6.3}$. Aunque ninguna de las peticiones cursadas contó con la aprobación re-

58. Gaceta de Madrid, 30 de enero de 1776.

59. A.G.S. Gracia y Justicia. Leg. 150. El marqués de la Ensenada a Alonso Muñiz. Buen Retiro, 29 de febrero de 1749; y Gaceta de Madrid, 18 de marzo de 1749.

60. A.H.N. Consejos. Leg. 18.543. Corregimiento de Cervera, 1765; y Gaceta de Madrid, 18 de junio de 1765.

61. A.H.N. Consejos. Leg. 18.552. Toma de posesión del correginiento de Talam, 1762.

62. A.G.S. Guerra Moderna. Leg. 1.965. Instancia del Brigadier D. Antonio Labeli. Talarn, 25 de mayo de 1765 .

63. A.G.S. Guerra Moderna. Leg. 1.965. Memorial de D. Jerónimo Gentile, 1766. 
gia, como le ocurriera también al capitán Domicio, a quien nos referimos anteriormente, a Gentile se le confió el empleo de corregidor, si bien en un destino distinto al solicitado, en este caso el de Teruel ${ }^{64}$. Gentile dispuso, además, de una considerable ventaja al no tener que desplazarse hasta la Corte a prestar el obligado juramento, ya que una real cédula, fechada el 11 de marzo de 1766, le facultaba para hacerlo ante el Real Acuerdo de la Audiencia aragonesa ${ }^{65}$.

Una vez tomada la correspondiente posesión del cargo, Gentile permaneció en el mismo hasta que le sobrevino la muerte, el primero de agosto de 1775.

También encontró acomodo en tierras aragonesas Nicolás Cavaseliche, un militar italiano que desde 1703 venía prestando sus servicios en favor del primer borbón español ${ }^{60}$.

Tras participar en la Guerra de Sucesión y en las campañas italianas, Cavaseliche, que desde 1716 contaba con el título de marqués de su apellido ${ }^{67}$, fue designado corregidor de Cinco Villas en 1721, empleo que desempeñó durante un trienio, cesando en julio de 1724. Alejado temporalmente de Aragón, cinco años más tarde volvía para hacerse cargo del mismo destino ${ }^{68}$, si bien esta vez su presencia al frente del corregimiento se vio seriamente comprometida al cabo de un tiempo al ser sometido a investigación como consecuencia de ciertas irregularidades observadas.

El 12 de abril de 1734 se puso una querella de 35 capítulos a Cavaseliche en la que se formulaban acusaciones muy graves. Sometido a pesquisa, el juez le condenó a suspensión del oficio de corregidor y multa de 1.000 ducados, sentencia que el Consejo dejó sin efecto a condición de que no volviese a ejercer la jurisdicción ordinaria en las Cinco Villas.

La pena impuesta suponía un grave atentado contra el honor del marqués, de ahí que éste recurriera al monarca alegando que:

«... si bastase sólo el ser acusado por constituirse reo, sería merecedor de que V.M. lo castigase, pero justificándose inocente, no tan solamente no es digno de pena, antes de premio ${ }^{69}$.

Las quejas de Cavaseliche, y sus tentativas por conseguir que el corregimiento de Cinco Villas no fuera provisto en otro sujeto en tanto no se le daba a él mismo el correspondiente acomodo en otro empleo, no tuvieron el menor éxito ${ }^{70}$. El fracaso de

64. A.G.S. Guerra Moderna. Leg. 1.965. Real Decreto confiriendo el corregimiento de Teruel. El Pardo, 15 de febrero de 1766; y en Gaceta de Madrid, 4 de marzo de 1766.

65. A.H.N. Consejos. Lib. 2.283, ff. 237-237v.

66. La trayectoria de Cavaseliche al frente del corregimiento de Cinco Villas ha sido estudiada por GIMÉNEZ LÓPEZ, E., «Los corregimientos de capa y espada como retiro de militares. El ejemplo de las Cinco Villas de Aragón en el siglo XVIII», en Revista de Historia Jerónimo Zurita 63-64 (1994), pp. $171-189$.

67. Gaceta de Madrid, 28 de enero de 1716; y ATIENZA, J., Nobiliario español, pp. 1.440-1.441.

68. A.G.S. Gracia y Justicia. Lib. 1.566.

69. A.G.S. Guerra Moderna. Leg. 1.899. Memorial del Marqués de Cavaseliche, 1739.

70. En un memorial redactado en 1739 reconocía el marqués que había «sido capitulado en el Consejo, y después de haber padecido cinco años esta injusta persecución, fuera de su empleo, sin sueldo, y sufriendo tan imponderables gastos en seguimiento de este pleito, lo declara el Consejo inocente sin que le haya condenado ni a restituir un maravedís a nadie, ni aun simple apercibimiento, y sólo consulta a V.M. de que le conmute su empleo atendiéndole en otro por los inconvenientes [que] podrian resultar, 
la posición mantenida se puso en evidencia cuando en 1742 el coronel Antonio Ruiz Sabeli fue designado para el referido corregimiento aragonés ${ }^{71}$.

Desde que su actuación en Cinco Villas empezó a ser cuestionada, Cavaseliche buscó, sin fortuna, un nuevo destino en el que instalarse. En 1736 pidió el gobierno de Cartagena ${ }^{12}$, mientras cuatro años más tarde optaba por los de Alcántara o Zamora, a la vez que solicitaba su ascenso a brigadier. En apoyo de semejantes pretensiones, Cavaseliche no dudó en recurrir a los méritos contraídos durante la Guerra de Sucesión, en los comienzos de la cual «vistió y armó un regimiento a su costa, con el cual pasó desde Nápoles a Barcelona; que sin embargo de hallarse sitiada se introdujo en ella a vista de los enemigos, que lo atacaron cerca de Mataró». También recordó haber «sacrificado gustoso su hacienda en el reino de Nápoles, donde por conservar la debida fidelidad a V.M. padeció por espacio de diez meses una dura prisión, puesto en un calabozo en el castillo nuevo de dicha ciudad, sin contar los demás rigores que experimentó con los demás prisioneros en poder de los alemanes, sin haber sido asistido en cosa alguna» ${ }^{73}$.

Pese al negativo resultado de las gestiones realizadas por Nicolás Cavaseliche para obtener un nuevo destino, el pronto fallecimiento de Ruiz Sabeli, ocurrido en 1745, y la rehabilitación de que fue objeto el militar italiano, que ese mismo año fue absuelto y declarado buen ministro, le colocaron nuevamente al frente del corregimiento de Cinco Villas ${ }^{74}$, al que se incorporó el 30 de junio de 1745 y en el que se mantuvo hasta su fallecimiento en 1754.

Dedicado a la carrera militar desde 1679, Blas Dragonetti, marqués de Dragonetti, era un napolitano que, como tantos otros compatriotas, apoyó la causa de Felipe V durante la Guerra de Sucesión española y fue recompensado por ello con un puesto en la administración territorial hispana. Sabemos que fue gobernador de Badajoz hasta 1715, año en que pasó a ocupar el corregimiento de Ciudad Rodrigo ${ }^{75}$.

Su estancia en el destino mirobrigense debió resultar muy satisfactoria para el marqués de Dragonetti, pues poco después de ser ascendido a teniente general, a mediados de 1720 , solicitó la concesión de una prórroga en atención a sus «dilatados servicios, graves achaques y crecida familia» ${ }^{76}$.

El deseo del marqués no fue contradicho por ninguno de los personajes que, tras ser preguntados, informaron sobre su conducta y cualidades personales, si bien algunas de las frases vertidas por el obispo de Salamanca pudieron influir en el negativo resultado de la pretensión. El gobernador del Consejo, Luis de Miraval, fue especial-

y cuando esperaba de que la benignidad de V.M., atendiendo a su inocencia, a sus atrasos, a sus servicios, y a lo que injustamente ha padecido en cinco años, le diese empleo correspondiente, como lo ha pedido, experimenta que en perjuicio de su honra, y derogándose el Real Decreto de V.M., del que hace ostentión (sic), se consulta su empleo antes que V.M. le dé el correspondiente que tiene mandado por dicho Real Decreto».

71. A.G.S. Gracia y Justicia. Leg. 148. Decreto de 7 de febrero de $I 742$.

72. A.G.S. Guerra Moderna. Leg. 1.886.

73. A.G.S. Guerra Modema. Leg. 1.905. Memorial.

74. A.H.N. Consejos. Leg. 18.014. Sobre el corregimiento de las Cinco Villas de Aragón.

75. Gaceta de Madrid, 26 de febrero de 1715.

76. A.G.S. Guerra Moderna. Leg. 1.855. El marqués Dragonette al marqués de Tolosa. Ciudad Rodrigo, 19 de octubre de 1720 . 
mente generoso en su juicio al indicar que «se ha portado muy bien, desempeñando su obligación en cuanto se le ha encargado, y mantenido aquella ciudad en toda

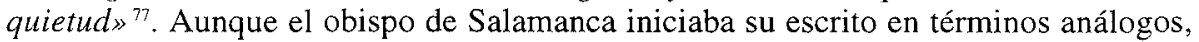
no por ello dejaba de reconocer los negativos efectos que el paso del tiempo había operado sobre el otrora brillante militar:

«se me ha asegurado que en cuanto está de su parte ha procurado el más exacto cumplimiento, pero que hallándose ya con muy crecida edad, y por eso con menos fuerzas que aquellas de que necesita su empleo, fía mucha parte de él al alcalde mayor, quien resuelve las dependencias con alguna intervención de la marquesa, cuya circunstancia no deja de desagradar a los interesados ${ }^{78}$.

Con el parecer del prelado salmantino, y el resultado del juicio de residencia a que fue sometido Dragonetti a partir de $1721^{79}$, tras concluir su labor en el corregimiento de Ciudad Rodrigo ${ }^{80}$, la continuidad del militar napolitano en este destino se hizo inviable, por lo que fue transferido a otra área geográfica, concretamente Cataluña.

Designado corregidor de Tarragona en 1724 para cubrir la vacante existente por fallecimiento de Tiberio Carraffa, Dragonetti tomó posesión del cargo el primer día de julio. Apenas unos meses más tarde, sin embargo, solicitaba su vuelta a Extremadura argumentando motivos de salud. La petición, que contó con el respaldo del conde de Montemar, fue rápidamente aceptada al señalar éste que:

«desde que se halla en Tarragona no ha dejado un instante la cama, y se le han agravado conocidamente sus achaques, con la evidente desconfianza de poder recobrar la salud mientras permaneciere en Tarragona, en cuya plaza le considera incapaz de servir, y aun expuesto a perderse muy en breve, por lo contraria que son el clima y la inmediación del mar a la enfermedad que padece» ${ }^{81}$.

De regreso a tierras extremeñas, el cambio de clima parece que contribuyó a que su salud se restableciera temporalmente, falleciendo en Badajoz el 18 de octubre de $1729^{82}$.

Como la mayor parte de los italianos que sirvieron empleos de gobierno en la península ibérica, también el coronel Esteban Smenota había dedicado buena parte de su vida a servir en el ejército cuando, a la altura de 1773 , fue agraciado con el gobierno militar y político de las Cuatro Villas de la Costa de la $\mathrm{Mar}^{83}$.

77. A.G.S. Guerra Moderna. Leg. 1.855. Luis de Miraval a Miguel Fernández Durán. Madrid, 28 de noviembre de 1720 .

78. A.G.S. Guerra Moderna. Leg. 1.855. Silvestre, obispo de Salamanca, a Miguel Fernández Durán. Salamanca, 11 de diciembre de 1720.

79. Durante el juicio de residencia a que fue sometido Blas Dragonetti en 1721 se le hicieron seis cargos, siendo condenado en 3.600 maravedíes por cinco de ellos, y absuelto en el sexto, en A.G.S. Guerra Moderna. Leg. 1.855. Autos de la residencia tomada al gobernador de Ciudad Rodrigo Marqués de Dragonetti.

80. En tanto se le confiaba otro empleo, y mientras se desarrollaba el juicio de residencia, un decreto regio indicaba que «he resuelto que continuéis mi servicio en la provincia de Extremadura, en el paraje que os señale el Comandante General de ella", con sueldo de teniente general en cuartel. Cfr. A.G.S. Guerra Moderna. Leg. 1.855. Real Decreto dado en Madrid a 26 de noviembre de 1721.

81. A.G.S. Guerra Moderna. Leg. 1.859. El conde de Montemar. Barcelona, 23 de septiembre de 1724.

82. Gaceta de Madrid, 1 de noviembre de 1729.

83. Gaceta de Madrid, 16 de febrero de 1773. 
Si los individuos a que venimos haciendo referencia sirvieron sus empleos en propiedad, también los hubo que debieron contentarse con desempeñarlos con carácter interino, como sucedió a Agustín Doria. Teniente coronel del regimiento de infantería de Nápoles desde $1727^{84}$, y del regimiento de Milán en 1737, Doria pasó algo más de un año en Madrid -desde enero de 1734 hasta abril de 1735- proponiendo, sin éxito, la leva de un regimiento; dos años después marchó a San Felipe, en el reino de Valencia, donde desempeñó interinamente el corregimiento desde la salida del coronel Francisco Bie a la llegada de su sustituto, el también coronel Miguel Vandooren ${ }^{85}$.

Aunque aspirantes a un corregimiento, otros militares italianos no tuvieron la misma suerte que los compañeros a los que acabamos de referirnos: José Deu o Rodulfo Aquaviva y Aragón fueron algunos de ellos.

Natural de Cerdeña, los informes que se redactaron en 1715, cuando José Deu fue propuesto en segundo lugar, sin éxito, para el corregimiento de Cervera, ponían especial énfasis en su participación en la defensa y recuperación de la isla mediterránea ${ }^{8}$. Decía García Pérez Araciel:

«natural de Cerdeña, ha servido en aquella isla, así para conservarla como para recuperarla cuando se intentó, y también sirvió en el ejército de Portugal a S.M.» ${ }^{87}$.

En cuanto al resto de sus cualidades aseguraba Araciel que «por lo que toca a su capacidad he oído hablar muy bien y parece que su fidelidad es acreedora de la benignidad Real».

En el ejército desde 1709, el mariscal de campo Rodulfo Aquaviva había participado en numerosos episodios bélicos, como la expedición de Sicilia y el sitio de Gibraltar, cuando pretendió, allá por el año 1740, que se le confiara el corregimiento de Cádiz. El veterano militar pedía, asimismo, poder retener la tenencia de la compañía italiana de Guardias de Corps que se hallaba sirviendo, tal y conforme se permitió al marqués de Ruchena siendo ayudante general del mismo cuerpo ${ }^{88}$. Aunque el informe que el Cardenal Molina, Gobernador del Consejo por aquellas fechas, practicó sobre el aspirante ponía más énfasis en sus cualidades que en su falta de experiencia, Aquaviva no logró ser designado; decía el Cardenal que:

«aunque no está experimentado en gobiernos, demuestra viveza y espiritu, buen corazón, genio bizarro y desinteresado, cual corresponde a su notorio nacimiento, por lo que con la ayuda de un buen alcalde mayor -que los militares necesitan más que otros para el gobierno político- me parece desempeñará también las obligaciones de tal encargo» ${ }^{89}$.

Como los anteriores, también el sargento mayor del regimiento de infantería de Milán Alejandro Gaudino pretendió en 1721, sin éxito, el corregimiento de Castellón

84. Gaceta de Madrid, 19 de agosto de 1727.

85. GIMÉNEZ LÓPEZ, E., Militares en Valencia..., p. 203.

86. En este mismo sentido se manifestaba el marqués de Bedmar. Cfr. A.G.S. Gracia y Justicia. Leg. 134. Marqués de Bedmar a Manuel Vadillo y Velasco. Madrid, 19 de octubre de 1715.

87. A.G.S. Gracia y Justicia. Leg. 134. Informe de García Pérez de Araciel, 1715.

88. A.G.S. Guerra Moderna. Leg. 1.905. Memorial de Rodulfo Aquaviva pretendiendo el gobierno de Cadiz. 1740 .

89. A.G.S. Guerra Moderna. Leg. 1.905. El cardenal de Molina al marqués de Uztáriz. Madrid, 15 de abril de 1740 . 
de la Plana ${ }^{y 0}$. Aunque este primer intento acabó en fracaso, algunos años más tarde se le confió la tenencia de rey de Denia ${ }^{91}$.

Si buen número de oficiales italianos encontraron acomodo al frente de un corregimiento, sobre todo en tierras de la Corona de Aragón, también fue en este ámbito geográfico donde más se prodigaron aquéllos en la titularidad de un gobierno militar.

Lofredo Caetano, coronel del regimiento de infantería de Milán, ocupó el cargo de gobernador de Balaguer, en Cataluña, desde el verano de 1736 hasta 1739 en que falleció ${ }^{92}$.

Como Caetano, también Jorge Domingo Traggia Aliprandi desempeñó durante algún tiempo el gobierno balagariense. Nacido en 1674 en Mezzana de Rabaton, lugar del señorío de los Aliprandi, Jorge Domingo Traggia era un militar que dejó su Italia natal mediada la segunda década del Setecientos para establecerse en España, concretamente en Zaragoza, ciudad a la que llegó en $1717^{93}$.

Una vez afincado en Aragón, Jorge Domingo obtuvo permiso en el año 1719 para la creación de un regimiento de dragones, compuesto por 600 hombres, del que fue coronel y para el cual nombró a los oficiales, uno de los cuales fue su propio hijo Carlos Antonio, quien por aquél entonces se hallaba sirviendo en el ejército de Sicilia.

La dedicación de Traggia a la milicia se vio recompensada cuando Felipe $\mathrm{V}$ le confió el gobierno de Balaguer, en Cataluña, en la primavera de $1727^{\text {भt }}$. No debió ser acogido, sin embargo, de muy buen grado este destino por el militar italiano, ya que transcurridos apenas seis meses Jorge Domingo se dirigía al Secretario del despacho de Guerra, Baltasar Patiño, solicitando su traslado a Monzón, plaza que acababa de quedar vacante al ser designado su titular, el mariscal de campo Blas de Trincheria, nuevo gobernador de Zaragoza. La principal razón que impulsaba a Traggia a pedir el cambio de destino era, según se podía leer en su escrito al marqués del Castelar, la mayor oferta cultural que allí podría encontrar para dar una buena educación a sus hijos:

«aseguro a V.E. sería uno de los mayores ascensos que podía lograr para el consuelo y educación de mis pobres hijos, por la conveniencia de tener en Barbastro (dos haras distantes de Monzón) un colegio de escolapios de los mejores que hay en este Reino, con que les podría dar la mejor educación y enseñanza, con otras ventajosas conveniencias que se me seguian."

El negativo resultado de la gestión realizada, y el amplio patrimonio que, con el paso del tiempo, fue adquiriendo Jorge Domingo en bienes raíces en Zaragoza y sus alrededores (casas, fincas, huertas, torres), le sirvieron de incentivo para tratar de conseguir un arraigo todavía mayor: lograr un puesto en el consistorio zaragozano.

Traggia, que había adquirido cierta experiencia al frente del gobierno de Balaguer, pretendió y logró un empleo de regidor en el ayuntamiento de la capital arago-

90. A.G.S. Guerra Moderna. Leg. 1.855. Memorial de Alejandro Gaudino, 1721.

91. Gaceta de Madrid, 29 de abril de 1727.

92. Gaceta de Madrid, 14 de agosto de 1736; y A.G.S. Guerra Moderna. Leg. 1899.

93. ARIJA NAVARRO, M.A., Joaquín Traggia y su tiempo: el concilio provincial de Manila de 1771 . Tesis doctoral inédita, Departamento de Historia Moderna, Universidad de Zaragoza, 1982, ff. 81-88.

94. Gaceta de Madrid, 15 de abril de 1727.

95. A.G.S. Guema Moderna. Leg. 1.866. Domingo Traggia al marqués de Castelar. Zaragoza, 9 de septiembre de 1727. 
nesa a finales de 1728 , al ser designado para cubrir la vacante ocurrida por fallecimiento de Pedro Crel.

Aunque en un principio el resto de sus compañeros pusieron reparos a su incorporación basándose en su origen italiano, en una Real Cédula fechada el 14 de enero de 1729 Felipe $\mathrm{V}$ le dispensó de su condición de extranjero, de ahí que pasara acto seguido a ejercer el cargo sin más contradicciones.

Jorge Domingo fue el primer Traggia en obtener una regiduría en el ayuntamiento zaragozano, una experiencia que debió ser valorada muy positivamente por los miembros de esta familia, ya que tras él, tanto su hijo Domingo, como su nieto José Joaquín, desempeñaron, sucesivamente, idéntica función ${ }^{46}$.

Domingo Traggia Roncal tomó posesión del regimiento zaragozano en marzo de 1736, apenas dos meses antes de que se produjera el fallecimiento de su padre, quien previamente había renunciado dicho empleo en su favor. Cuando, transcurridas casi dos décadas, Domingo decidió abandonar el ayuntamiento para ingresar en religión ${ }^{97}$, utilizó la misma práctica que su progenitor, cediendo esta vez el oficio a su hijo José Joaquín.

La incorporación de José Joaquín Traggia al consistorio zaragozano coincidió, prácticamente, con su agregación al ejército, empleos que difícilmente podría desempeñar de manera simultánea. Si como regidor fue el encargado de gestionar el alojamiento de Carlos III en la ciudad cuando éste regresó de Nápoles, es sobre todo su faceta militar la que merece especial atención.

Cadete de Reales Guardias Españolas desde 1754, José Joaquín Traggia practicó en sus comienzos «algunas salidas en Tarragona a los moros»; participó en la Guerra de Portugal, en 1761, con la Compañía de Voluntarios de Caballería; y desde 1768, año en que obtuvo el grado de coronel, hasta 1787 permaneció como comandante de la Compañía de Voluntarios de Andalucía, también de caballería, destacando en la persecución de contrabandistas y malhechores.

Tras un cuarto de siglo dedicado a lograr la pacificación del solar hispano, ya fuera combatiendo a otros ejércitos, o a los piratas y bandidos que inquietaban a la población civil, José Joaquín pretendió, si bien sin éxito, el corregimiento de las Cinco Villas ${ }^{\text {. }}$.

El resultado negativo de sus gestiones no desanimó a Traggia, quien cinco años más tarde, en 1784, se mostraba aspirante a la Tenencia de Rey de Zaragoza ${ }^{94}$; tampoco esta vez logró su objetivo.

Dispuesto a no cejar en su empeño de lograr un puesto de mando, en 1787, y tras producirse el fallecimiento de Francisco Javier Jacome, Traggia solicitó nuevamente el corregimiento de las Cinco Villas, en Aragón. Como argumentos de peso que pu-

96. Sobre la presencia de los Traggia en el consistorio zaragozano vid. el artículo de MORENO NIEVES, J.A., «Los militares en el gobierno local aragonés tras el decreto de Nueva Planta», en este mismo número.

97. Domingo Traggia recibió la tonsura el 17 de febrero de 1755 , ordenándose de diácono y presbítero en julio siguiente; tres años más tarde, el 4 de abril de 1758, profesaba como carmelita descalzo. Cfr. ARIJA NAVARRO, M.A., Joaquín Traggia..., ff. 117-118.

98. A.G.S. Guerra Moderna. Leg. 1.966. Pretendientes al Gobierno de Cinco Villas, 1779.

99. A.G.S. Guerra Moderna. Leg. 1.967. Tenencia de Rey de Zaragoza y Gobierno del Castillo de la Aljaferia, 1784 
dieran inclinar al monarca a confiarle el cargo apetecido señalaba que «a imitación de toda su familia sirve a V.M. 33 años há (...), y por la mucha familia con que se halla se ve precisado a suplicar a la piedad de V.M.... ${ }^{100}$.

Logrado esta vez el fin perseguido, Traggia tomó posesión del empleo a primeros del mes de junio en la villa de Sos, donde residía la capitalidad de las Cinco Villas. Sin embargo, no transcurrió mucho tiempo sin que José Joaquín recurriera nuevamente al monarca solicitando esta vez permutar el corregimiento del que era titular por el de Huesca; de accederse a su petición podría ocuparse personalmente de una hacienda que poseía en el referido distrito y, con su atención directa, mejorar su rendimiento "para alivio de su dilatada familia» ${ }^{101}$.

Puesto que Traggia no consiguió el apetecido traslado a Huesca, y sabedor de que Cinco Villas era una especie de cementerio al que iban a terminar su carrera militares de edad provecta ${ }^{102}$, en 1799, afectado por graves dolencias, decidió dejar el manejo del corregimiento en manos del teniente coronel Ignacio Asensio -hasta ese momento gobernador de Monzón-, si bien con la condición de seguir cobrando su sueldo mientras viviera ${ }^{103}$. Esta vez sí logró el veterano militar, que había sido ascendido a brigadier cinco años antes, su recompensa a toda una vida de dedicación al real servició, al condescender el rey con la petición cursada ${ }^{104}$.

José Joaquín no fue el único de los hermanos Traggia Uribarri que dedicó su vida al ejercicio de las armas, ya que también Domingo Mariano se incorporó desde muy joven al ejército, concretamente al cuerpo de caballería. Siguiendo la tradición familiar también figuró al frente de un gobierno a partir de 1790 , en este caso el de Río Alhama ${ }^{105}$.

La trayectoria de la familia Traggia supone un claro ejemplo de asimilación a la oligarquía local, en este caso zaragozana, así como de integración en la realidad político-social hispana; buena prueba de ello es, por ejemplo, que tanto Claudio Traggia, como sus sobrinos José Joaquín, Domingo Mariano y Felipe, se incorporaron como caballeros de la orden de Santiago en $1769^{106}$.

Para cerrar la nómina de individuos que venidos de la península vecina asumieron algún gobierno en tierras hispanas citaremos al conde de Bolognino, quien fue destinado a Cartagena a finales de 1760 , cuando contaba el grado de mariscal de campo ${ }^{107}$. Unos años más tarde, en 1767 , le fue confiada la comandancia General de la Plaza de Orán ${ }^{108}$, siendo posteriormente ascendido a teniente general ${ }^{109}$.

100. A.G.S. Guerra Moderna. Leg. 1.968. Memorial de D. José Traggia solicitando el corregimiento de las Cinco Villas. Madrid, 5 de marzo de 1787.

101. A.G.S. Guerra Moderna. Leg. 1.968. Memorial de D. José Traggia. Sos, 13 de noviembre de 1787.

102. GIMÉNEZ LÓPEZ, E., «Los corregimientos de capa y espada como retiro de militares...», pp. 171189.

103. A.G.S. Guerra Moderna. Leg. 6.370. Memorial de José Traggia e Ignacio Asensio. Zaragoza, 15 de junio de 1799.

104. Gaceta de Madrid, 12 de julio de 1799, p. 625.

105. Gaceta de Madrid, 26 de octubre de 1790.

106. CADENAS Y VICENT, V., Caballeros de la orden de Santiago, vol. V, p. 167-168.

107. Gaceta de Madrid, 28 de octubre de 1760.

108. Gaceta de Madrid, 21 de julio de 1767.

109. Gaceta de Madrid, 3 de abril de 1770. 
Aunque ya lo apuntamos al inicio del trabajo, no podemos dejar de reconocer, a modo de conclusión, que este artículo no es sino una aproximación a una realidad mucho más compleja, la de aquellos italianos que sirvieron a los monarcas hispanos del Setecientos, no sólo desde unos empleos con alcance geográfico limitado, como los de capitán general, intendente, corregidor o gobernador; sino también desde puestos en los que, como las Secretarías de Estado o Consejos, se tomaban decisiones que afectaban a toda la monarquía.

Sin embargo, ciñéndonos al tema abordado hay una serie de notas que merecen ser puestas de relieve, como la íntima vinculación que mantenían con el ejército la práctica totalidad de los individuos objeto de estudio. Otro aspecto no menos importante atañe a los enlaces matrimoniales que frecuentemente establecieron con notorias familias peninsulares; uniones que contribuyeron a que se produjera no sólo el arraigo de los individuos en cuestión, sino también de sus descendientes, que permanecieron en España y siguieron al servicio de la monarquía, como se percibe en los casos de los marqueses de Vallesantoro o los Traggia.

Una última cuestión a remarcar sería la de las distinciones honoríficas que alcanzaron, bien por méritos propios, bien condicionadas por los enlaces familiares establecidos: entrada en diferentes órdenes militares, obtención de cargos palaciegos y títulos nobiliarios, etc. 